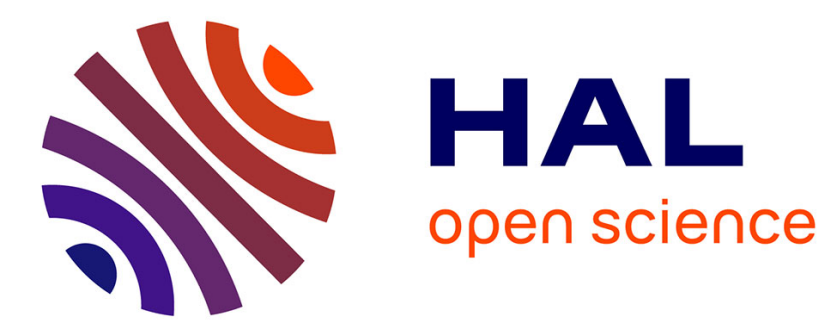

\title{
Dynamics in pure and substituted volborthite kagome-like compounds
}

F. Bert, P. Mendels, D. Bono, A. Olariu, F. Ladieu, J.-C. Trombe, F. Duc, C. Baines, A. Amato, A. Hillier

\section{- To cite this version:}

F. Bert, P. Mendels, D. Bono, A. Olariu, F. Ladieu, et al.. Dynamics in pure and substituted volborthite kagome-like compounds. Physica B: Condensed Matter, 2006, 374, pp.134 - 137. 10.1016/j.physb.2005.11.034 cea-01396624

\section{HAL Id: cea-01396624 https://hal-cea.archives-ouvertes.fr/cea-01396624}

Submitted on 14 Nov 2016

HAL is a multi-disciplinary open access archive for the deposit and dissemination of scientific research documents, whether they are published or not. The documents may come from teaching and research institutions in France or abroad, or from public or private research centers.
L'archive ouverte pluridisciplinaire HAL, est destinée au dépôt et à la diffusion de documents scientifiques de niveau recherche, publiés ou non, émanant des établissements d'enseignement et de recherche français ou étrangers, des laboratoires publics ou privés. 


\title{
Dynamics in pure and substituted volborthite kagome-like compounds
}

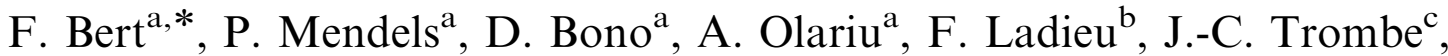 \\ F. Duc ${ }^{\text {c }}$, C. Baines ${ }^{\mathrm{d}}$, A. Amato ${ }^{\mathrm{d}}$, A. Hillier ${ }^{\mathrm{e}}$ \\ ${ }^{a}$ Laboratoire de Physique des Solides, UMR 8502, Université Paris-Sud, 91405 Orsay, France \\ ${ }^{\mathrm{b}}$ Service de Physique de l'État Condensé, DSM, CEA Saclay, 91191 Gif-sur-Yvette Cedex, France \\ ${ }^{\mathrm{c}}$ Centre d'Élaboration des Matériaux et d'Études Structurales, CNRS UPR 8011, 31055 Toulouse, France \\ ${ }^{\mathrm{d}}$ Paul Scherrer Institut, Laboratory for Muon Spin Spectroscopy, CH-5232 Villigen PSI, Switzerland \\ ${ }^{\mathrm{e}}$ ISIS Facility, CLRC Rutherford Appleton Laboratory Chilton, Didcot, Oxon OX11 0QX, UK
}

\begin{abstract}
Volborthite $\mathrm{Cu}_{3} \mathrm{~V}_{2} \mathrm{O}_{7}(\mathrm{OH})_{2} \cdot 2 \mathrm{H}_{2} \mathrm{O}$ is a natural frustrated antiferromagnet with a kagomé-like magnetic lattice decorated by $S=\frac{1}{2}$ spins. Our $\mu$ SR results confirm the existence of a dynamical plateau below $2 \mathrm{~K}$, as initially demonstrated in [Phys. Rev. Lett. 91 (2003) 207603] despite sensible quantitative differences in the relaxation rate values. Thanks to additional low temperature SQUID and NMR data we show that this plateau occurs concurrently with a freezing transition. Moreover, we revisited the effect of diluting the magnetic lattice for small dilution rates between $1 \%$ and $15 \%$. Comparison is made with the well studied kagomé bilayer compounds. The ground state of volborthite appears to be far less stable with respect to dilution.
\end{abstract}

(C) 2005 Elsevier B.V. All rights reserved.

PACS: 75.50.Lk; 76.75. $+\mathrm{i} ; 76.60 .-\mathrm{k}$

Keywords: Muon-spin rotation; Kagomé lattice; Frustrated magnets

In the field of frustrated antiferromagnets, the recently rediscovered volborthite $\mathrm{Cu}_{3} \mathrm{~V}_{2} \mathrm{O}_{7}(\mathrm{OH})_{2} \cdot 2 \mathrm{H}_{2} \mathrm{O}[1,2]$ plays a special role as it approaches the theoretical model of quantum spins $S=\frac{1}{2}$ on the highly frustrated kagomé lattice. For such a spin system, the ground state is expected to be of liquid type, with short range correlations and persisting dynamics at $T \rightarrow 0[3,4]$.

Seminal muon spin relaxation experiments demonstrated the existence of persisting dynamics in the archetypal $\mathrm{Cr}^{3+}$ $\left(S=\frac{3}{2}\right)$ kagomé bilayers $\mathrm{SrCr}_{9 p} \mathrm{Ga}_{12-9 p} \mathrm{O}_{19}(\mathrm{SCGO})[5]$ and $\mathrm{Ba}_{2} \mathrm{Sn}_{2} \mathrm{ZnGa}_{10-7 p} \mathrm{Cr}_{7 p} \mathrm{O}_{22}[6](p<0.97)$ or the $\mathrm{Cr}^{3+}$ jarosite compounds [7]. However direct comparison to theoretical calculation still claims for a closer experimental realization of the theoretical model. Moreover understanding the interplay between the persistent dynamics observed in $\mu \mathrm{SR}$ and the concurrent freezing observed at low- $T$ in susceptibility measurements in the archetypal frustrated magnets

\footnotetext{
${ }^{*}$ Corresponding author. Fax: + 33169156086

E-mail address: bert@lps.u-psud.fr (F. Bert).
}

[8] remains a pending question and certainly deserves further theoretical and experimental investigations on new systems.

Volborthite consists of $\mathrm{CuO}$ layers well separated from each other by $\mathrm{V}_{2} \mathrm{O}_{7}$ pillars and water molecules. In this compound, $\mathrm{Cu}^{2+}\left(S=\frac{1}{2}\right)$ are the only magnetic atoms and they form planes of corner-sharing triangles. The resulting magnetic structure is a stacking of well separated $(7.2 \AA)$ kagomé-like lattices fully covered by $S=\frac{1}{2}$ spins. Due to a small monoclinic distortion, there might exist two $\mathrm{Cu}-\mathrm{Cu}$ interaction constants, i.e. the kagomé lattice is built on isosceles triangles rather than equilateral ones. Whether this possible dissymmetry of the exchange paths impacts the nature of the ground state (GS) is still an open question and certainly requires refined calculation. On experimental grounds, a former $\mu \mathrm{SR}$ study [9] evidenced a slowing down of spin fluctuations below $5 \mathrm{~K}$ followed by nearly temperature independent fluctuations below $1.5 \mathrm{~K}$ and down to $50 \mathrm{mK}$, demonstrating the liquid nature of the GS. The lowest temperature of former SQUID, heat 
capacity and NMR experiments [2] was $1.8 \mathrm{~K}$ and could not conclude on the nature of the low- $T$ regime exhibited in $\mu \mathrm{SR}$, in particular no magnetic transition was found above $1.8 \mathrm{~K}$. In order to investigate this low energy regime, as compared to the exchange energy $J \simeq 90 \mathrm{~K}$ [2], we performed low- $T$ SQUID, NMR and $\mu$ SR experiments on the pure compound. Besides, we investigated through $\mu \mathrm{SR}$ and SQUID measurements the effect of diluting the magnetic network of volborthite in $\mathrm{Zn}(S=0)$ substituted samples.

The macroscopic susceptibility of pure volborthite is presented in Fig. 1. At high temperature, the behavior is of the Curie-Weiss type with $\theta_{\mathrm{w}} \simeq 130 \mathrm{~K}$ but due to frustration no transition in the magnetic susceptibility is detected at this mean field temperature. The bump at around $25 \mathrm{~K}$ probably reflects the development of short range spin correlation at this temperature $[2,10]$. Below $2 \mathrm{~K}$ the separation of the zero field cooled (ZFC) and field cooled (FC) magnetization signals a freezing process. Below $2 \mathrm{~K}$, the $\mathrm{ZFC}$ branch shows a very broad maximum at around $1.3 \mathrm{~K}$ which probably arises from a rather large distribution of freezing temperatures as confirmed by NMR data [11].

Fig. 2 shows the muon relaxation rate in volborthite measured in a $100 G$ longitudinal applied field to decouple the nuclear dipoles. It appeared that the time dependence of the asymmetry cannot be fitted by a simple functional form on the whole time scale. The relaxation rates were estimated by taking into account the $1 / e$ decay time. As in Ref. [9], we find that the spin dynamics slow down below $5 \mathrm{~K}$ and reach a dynamical plateau below $2 \mathrm{~K}$. Despite qualitative agreement, the relaxation rate we measured appears to be 14 times larger than the one reported in Ref. [9]. Since defects in the magnetic structure reduce drastically the $T \rightarrow 0$ relaxation rate (see discussion on the $\mathrm{Zn}$ substituted sample), one possibility is that our samples

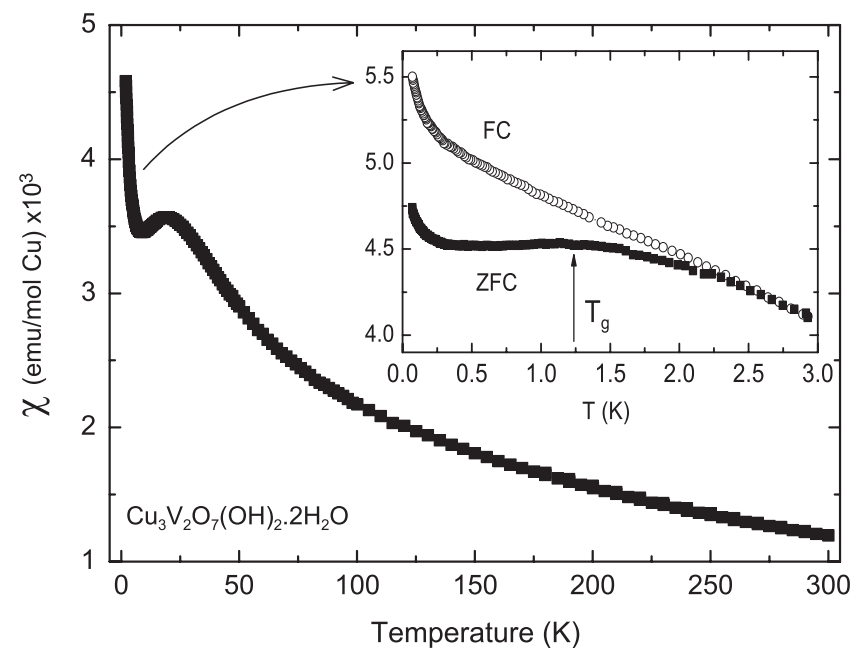

Fig. 1. Macroscopic susceptibility versus temperature in pure volborthite in 100 Oe. Inset: zoom on the very low temperature data. The upturn below $T \simeq 200 \mathrm{mK}$ is an artifact due to few ppm magnetic impurities in the grease used to ensure thermalization of the powder sample.

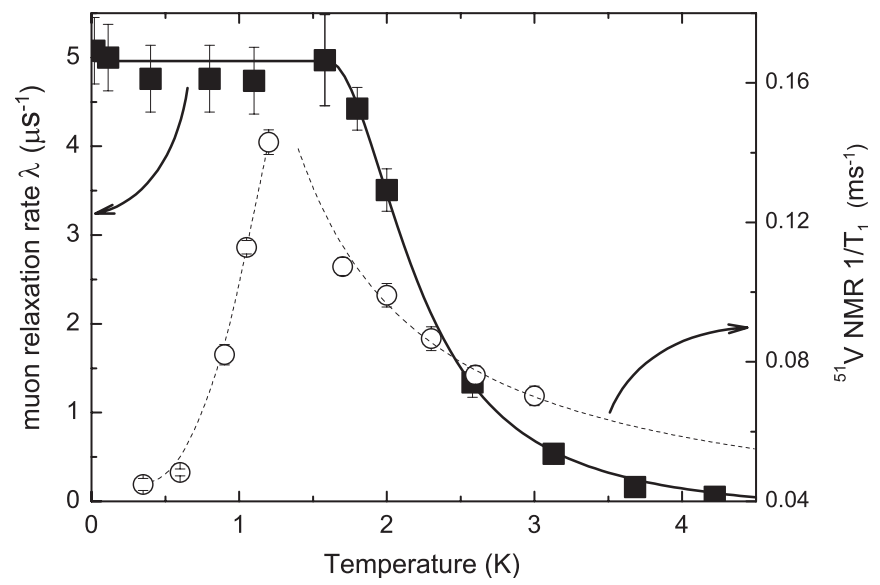

Fig. 2. Comparison of the $T$ dependence of the muon relaxation rate (left axis) and ${ }^{51} \mathrm{~V}$ nuclei relaxation rate in pure volborthite.

are slightly better crystallized as a result of a slightly different synthesis protocol (see details of preparation in Ref. [11]).

For comparison, we also plotted in Fig. 2 the vanadium nuclear spin lattice relaxation rate $\left(1 / T_{1}^{\mathrm{NMR}}\right)$ measured by pulsed NMR. Contrary to the muon relaxation rate, the latter decreases below the transition observed in the SQUID experiment, as expected for a frozen ground state. Moreover, if we assume that the field at the muon site is $H_{4}=35 G$ as in Ref. [9], from the muon relaxation we measured, we get $3.5 \mu \mathrm{s}^{-1}$ for the copper electronic spin fluctuations. From this value and the vanadium hyperfine coupling value $A=7.7 \mathrm{kOe}$, we would expect to get $1 / T_{1}^{\mathrm{NMR}} \simeq 20 \mathrm{~ms}^{-1}$, two orders of magnitude larger than what we measured. This suggests that the electronic spin fluctuations which are efficiently probed in $\mu \mathrm{SR}$ experiments are somehow filtered out at the vanadium nuclear site. Actually, the vanadium position in the unit cell is quite specific since it lays at the center of the hexagons of the kagomé lattice. Therefore for some very specific spin configurations and specific correlated fluctuations of the spins belonging to a same hexagon, it is possible that vanadium nuclei indeed only feel a static resulting field [11]. On the other hand, muons, which probably sit near oxygens, at non-symmetric sites, do not present this filtering effect.

In order to test the stability of the volborthite ground state with respect to dilution and to compare with the SCGO kagomé bilayer archetype, it is possible to substitute $\mathrm{Zn}^{2+}(S=0)$ at the $\mathrm{Cu}^{2+}$ site with no major distortion of the lattice. Decoupling experiment at base temperature in a $2.5 \%$ at. $\mathrm{Zn} / \mathrm{Cu}$ substituted sample is shown in Fig. 3. A small applied field of $65 G$ decouples the nuclear dipoles. Then for increasing fields, the decoupling is much less efficient. Asymmetry is not fully restored in a $1600 G$ field whereas the internal field at the muon site is of the order of $35 G$ as mentioned before [9]. Therefore the ground state in the substituted samples remains dynamical as for the pure 


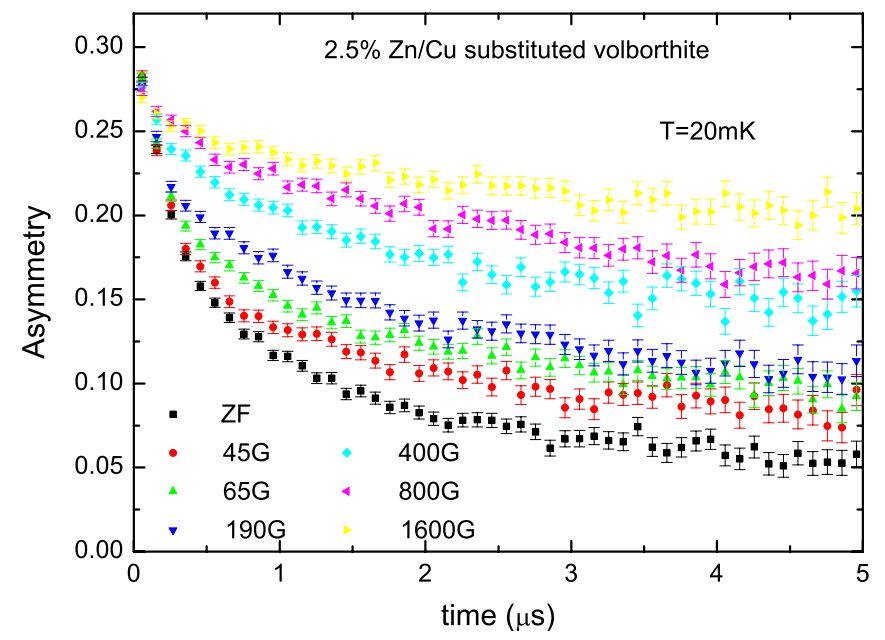

Fig. 3. Field dependence of the $\mu^{+}$disintegration asymmetry at base temperature $T=20 \mathrm{mK}$ in $2.5 \% \mathrm{Zn} / \mathrm{Cu}$ substituted volborthite.

compound, at least for small dilution rates. It is however noticeable that the relaxation rate at base temperature in this substituted sample $\left(\lambda \simeq 1 \mu \mathrm{s}^{-1}\right)$ is nearly five times smaller than in the pure sample.

Such a decrease of the relaxation rate on the dynamical plateau upon dilution was reported for kagomé bilayer [7,6] as well as for volborthite [9]. However, the decrease reported in Ref. [9] was only a factor 1.5 from the pure compound to the $5 \% \mathrm{Zn}$ substituted sample. To refine the more drastic effect of dilution we observed here, we studied systematically $\left(\mathrm{Cu}_{1-x} \mathrm{Zn}_{x}\right)_{3} \mathrm{~V}_{2} \mathrm{O}_{7}(\mathrm{OH})_{2} \cdot 2 \mathrm{H}_{2} \mathrm{O}$ samples for smaller dilution rates $x=0.01,0.025,0.05,0.1$ and 0.15 than in Ref. [9].

For all samples we found a low- $T$ dynamical plateau feature and the values of the relaxation rates on the plateau are presented in Fig. 4 as a function of the magnetic lattice covering. In comparison to the SCGO case, we find a much stronger effect of dilution of the magnetic network, even if we normalize the covering to the percolation threshold of each lattice, $p_{\mathrm{c}}=0.5$ for the kagomé bilayer and $p_{\mathrm{c}}=0.65$ for the simple kagomé lattice of volborthite [12]. If we assume that the existence of a well defined dynamical plateau is the signature of the original fluctuating ground state in highly frustrated magnets, then it appears that dilution destroys the coherent ground state of volborthite much more efficiently that the one of the kagomé bilayer. Whether this effect results from the different geometries of the lattices or reflects a different nature of the ground state for $S=\frac{1}{2}$ and $S=\frac{3}{2}$ spin systems is an open question. It seems however reasonable that the kagomé bilayer allows to better accommodate defects that the truly two-dimensional kagomé one.

In order to investigate further this dilution effect, we also performed low- $T$ SQUID measurements on two substituted samples with $2.5 \%$ and $5 \%$ dilution rate respectively. Like the pure compound, both present a rather broad transition at low- $T$ and we defined the transition tempera-
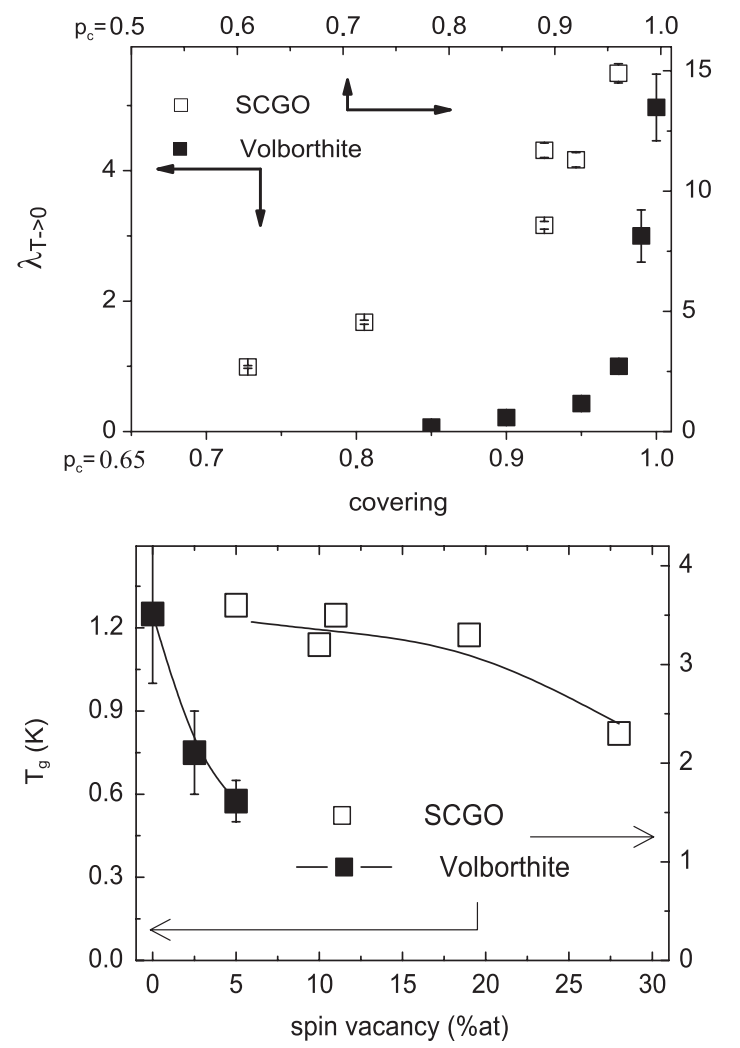

Fig. 4. Top panel: dependence on dilution of the relaxation rate on the dynamical plateau for a kagomé bilayer (SCGO) $[7,6]$ and volborthite (this study). The $x$ axes have been normalized by the percolation threshold for each lattice. Bottom panel: dependance on dilution of the transition temperature obtained by SQUID measurements [11,13] in the same compounds.

ture as the temperature of the maximum on the $\mathrm{ZFC}$ branch. The transition temperatures are shown in the bottom panel of Fig. 4 as a function of dilution and compared to SCGO compounds. Here again we find a much larger influence of dilution on the transition temperature than in SCGO. The simultaneous occurrence of the transition to a frozen state and the dynamical plateau observed in $\mu \mathrm{SR}$ in addition to the similar drastic effect of dilution on both the relaxation rate on the plateau and the transition temperature suggest a deep link between these two physical observations. Both seem to be manifestations of one original ground state.

In conclusion, with $\mu \mathrm{SR}$, NMR and low- $T$ SQUID experiments, we established that volborthite undergoes a transition to an original, partially frozen, state that still retains spin fluctuations. As a rather simple system, volborthite certainly opens route for close comparison to theoretical approaches.

This research project has been supported by the European Commission under Framework Programme 6 through the Key Action: Strengthening the European Research Area, Research Infrastructures. Contract no: RII3-CT-2003-505925. 


\section{References}

[1] M.A. Lafontaine, A.L. Bail, G. Férey, J. Solid State Chem. 85 (1990) 220.

[2] Z. Hiroi, M. Hanawa, N. Kobayashi, et al., J. Phys. Soc. Japan 70 (2001) 3377

[3] F. Mila, Phys. Rev. Lett. 81 (1998) 2356.

[4] G. Misguich, C. Lhuillier, Frustration in Two-Dimensional Quantum Antiferromagnets, World Scientific, 2003 cond-mat/0310405.

[5] Y. Uemura, A. Keren, K. Kojima, L.P. Le, et al., Phys. Rev. Lett. 73 (1994) 3306.

[6] D. Bono, P. Mendels, G. Collin, N. Blanchard, et al., Phys. Rev. Lett. 93 (2004) 187201
[7] A. Keren, K. Kojima, L. Le, G. Luke, et al., Phys. Rev. B 53 (1996) 6451.

[8] B. Martínez, F. Sandiumenge, A. Rouco, A. Labarta, et al., Phys. Rev. B 46 (1992) 10786.

[9] A. Fukaya, I.M. Gat, T. Ito, M.I. Larkin, et al., Phys. Rev. Lett. 91 (2003) 207603.

[10] F. Bert, D. Bono, P. Mendels, J.-C. Trombe, et al., J. Phys.: Cond. Matter 16 (2004) S829.

[11] F. Bert, D. Bono, P. Mendels, F. Ladieu, et al., Phys. Rev. Lett 95 (2005) 087203.

[12] C.L. Henley, Can. J. Phys. 79 (2001) 1307.

[13] L. Limot, P. Mendels, G. Collin, C. Mondelli, et al., Phys. Rev. B 65 (2002) 144447. 